\title{
Associations between the expression of micro-RNA 214 and clinicopathologic parameters of glioma
}

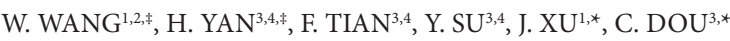 \\ ${ }^{1}$ The First Affiliated Hospital of Guangzhou Medical University, Guangzhou Medical University, Guangzhou 510120, China; ${ }^{2}$ Department of \\ Oncology, Inner Mongolia People's Hospital, Hohhot, 010052, China; ${ }^{3}$ Department of Neurosurgery, Nanfang Hospital, Southern Medical Univ- \\ ers ity, Guangzhou, 510515, China; ${ }^{4}$ Department of Neurosurgery, The Affiliated Hospital of Inner Mongolia Medical University, Inner Mongolia \\ Medical University, Hohhot, 010050, China
}

*Correspondence: dryhc1976@163.com,dryanhaicheng@163.com

*Contributed equally to this work.

Received December 18, 2013 / Accepted April 2, 2014

\begin{abstract}
It have been reported that miR-214 reduction facilitates UBC9 expression and is involved in the regulation of glioma cell proliferation. However, the specific role of miR-214 in glioma remains unknown. Thus, we investigated the relationship between expression level of miR-214 and clinico- pathological features and prognosis in patients with glioma in a follow-up of 5years. We used Chi-square tests for the categorical data and Mann-Whitney tests for continuous data. Survival time was calculated from the date of glioma diagnosis to the date of death or last follow-up. Survival analysis was estimated using the Kaplan-Meier method, log-rank test, and Cox-proportional hazards regression model. In the present study, we confirmed that the expression level of miR-214 was increased in glioma tissues compared with the non-neoplastic brain tissues. Next, the Kaplan-Meier analysis revealed that glioma patients with high miR-214 expression tend to have poorer overall survival. In addition, the multivariate analysis clearly demonstrated that high miR-214 expression was a statistically significant risk factor affecting overall survival in glioma patients, suggesting that miR-214 upregulation in gliomas is not only in a grade-dependent fashion, it is also a predictor of overall survival. Finally, subgroup analyses showed the significant prognostic value of miR-214 upregulation for glioma patients in those with low and high pathological grade. The results of this study showed that miR-214 was up-regulated in glioma tissues. The expression of miR-214 was associated with the pathological stages of glioma. The results of 5-years follow-up showed that the expression level of miR-214 is a significant prognostic factor for patients with glioma.
\end{abstract}

Key words: miR-214, glioma, prognosis

Brain and other nervous system tumors are, after stroke, the second cause of death from neurological diseases. Glioma is one of the most common malignant tumors, and it is the most common cancer in central nervous system of both children and adults [1]. The glioma was histologically classified as astrocytomas, oligodendrogliomas, and oligoastrocytomas [2]. These tumors are graded on the World Health Organization (WHO) grading system integrating four ascending grades of malignancy [3]. Moreover, because of our poor understanding of this cancer's pathogenesis, therapeutic strategies for

\footnotetext{
Abbreviations: WHO, World Health Organization; qRT-PCR, quantitative real-time polymerase chain reaction; miRNA, MicroRNAs, RISC, RNAinduced silencing complex; GSCs, glioma stem cells; CT, threshold cycle; DSS, disease-specific survival; RR, relative risk; UTR, untranslated region.
}

glioblastoma are limited [4]. To develop new therapeutic and preventive strategies for glioma, exploring the molecular mechanism involved in glioma and progression becomes an urgent demand for clinical work.

MicroRNAs are known as non-coding RNAs that regulate the expression of target mRNA These single-stranded RNAs are considered to play crucial roles in many normal cellular processes and the multistep processes of carcinogenesis $[5,6]$. The Sanger miRBase reports over 2000 miRNAs in the human genome and these miRNAs have been shown to regulate multiple hallmarks of cancer, for example, increased proliferation and evasion of cell death [7]. Conceptually, miRNAs may have either tumor suppressor or oncogene potential within cells if they target oncogenes or tumor suppressor genes, respectively. The first description of the role of miRNAs in tumorigenesis 
was the identification of their localisation in close proximity to chromosomal breakpoints [8]. Subsequent analysis revealed aberrant miRNA expression and function in many cancers, for example, miRNA-15a/16-1 and let-7 as tumor suppressors [9] and miRNA-17-92 cluster as oncogene [10].

Several miRNAs have been reported to be related with the development, progression and prognosis of glioma. For instance, miR-329 located on $14 \mathrm{q} 32.31$ is one of down-regulated miRNAs in glioma. In the analyses of nine patients with glioma and the LN18 and T98G cells, The E2F1 was identified as the target of miR-329. Overexpression of miR-329 blocked G1/S transition in LN18 and T98G cell lines, dramatically suppressed cell proliferation and the ability of colony formation. MiR-329 significantly decreased the phosphorylation levels of intracellular kinases Akt and expression of cyclin D1, but the expression of p21 was upregulated and cell growth was suppressed by inhibiting E2F1-mediated Akt pathway [11]. Lee HK et al [12] examined the expression of miR-145 in glial tumors and its function in glioma cells. Using TCGA analysis and real-time PCR we found that the expression of miR-145/143 cluster was downregulated in astrocytic tumors compared to normal brain specimens and in glioma cells and glioma stem cells (GSCs) compared to normal astrocytes and neural stem cells. Moreover, the low expression of both miR145 and miR-143 in glioblastomas was correlated with poor patient prognosis.

Derfoul A et al report that miR-214 expression is inversely correlated with Ezh2 mRNA and protein levels in MCF-7 and in the invasive MDA-MB-231 breast cancer cell line and that deletion of at least one copy of the miR-214 genomic alleles occurs in $24 \%(6 / 25)$ cases of breast cancer examined. Overexpression of miR-214, but not that of a mutant miR-214 version, results in inhibition of breast cancer cell proliferation and invasion [13]. It have been reported that miR-214 reduction facilitates UBC9 expression and is involved in the regulation of glioma cell proliferation [14]. However, the specific role of miR-214 in glioma remains unknown. Thus, we investigated the relationship between expression level of miR-214 and clinico-pathological features and prognosis in patients with glioma in a follow-up of 5years.

\section{Patients and methods}

Patients and specimens. This study was approved by the institutional review board of The First Affiliated Hospital of Guangzhou Medical University, Guangzhou Medical University, Guangzhou. Informed consent was obtained from all subjects.

Since July 2000, One hundred and seventy five human glioma tissue samples for qRT-PCR were obtained from Department of Neurosurgery, The First Affiliated Hospital of Guangzhou Medical University, Guangzhou Medical University, Guangzhou. All the patients had histopathologically confirmed glioma, without a prior history of other cancers. The non-tumor tissues were confirmed as such by an experienced pathologist and used as controls. Patients' characteristics and clinical features are recorded respectively. Tumors were staged using the WHO classification [2] by two pathologists, with differences resolved by careful discussion.

Samples were quickly removed at surgery and immediately divided into two parts; one part was fixed in $4 \%$ paraformaldehyde for 24 hours, paraffin embedded, and used for histopathological diagnosis, and the remaining part was snap frozen in liquid nitrogen and maintained at $-80^{\circ} \mathrm{C}$ until used for RNA isolation. All the slides were reevaluated according to WHO classifications [2] by two pathologists, with differences resolved by careful discussion.

Follow-up. At our center, patients with glioma are routinely followed once every 3 to 6 mo in the first three years, regardless of the stage of their disease. Time to death is defined from the date of surgery. At each visit, patients undergo a medical history and physical examination. Imaging tests and other required examinations are performed when clinically indicated.

Real-time quantitative RT-PCR for detection of miR-214. Total RNA was isolated using TRIzol reagent (Invitrogen, Carlsbad, CA). For miRNA qRT-PCR, expression of mature miR-214 was analyzed by TaqMan miRNA Assay (Applied Biosystems, Foster City, CA, USA) under conditions defined by the supplier. The TaqMan probes and primers for human miR-214 and internal control U6 were purchased from Applied Biosystems. Amplification and analysis were performed on the ABI 7900 sequence detection system. All quantitations were normalized to the level of human U6 snRNA in the reaction. The comparative threshold cycle (CT) (DDCT) method, which compares differences in CT values between common reference RNA and target gene RNA, was used to obtain the relative fold changes in gene expression. The miR-214 primers for PCR were designed by GenePharma Co., Ltd (Shanghai, China). The results are expressed as mean \pm SD.

Statistical methods. All statistical analyses were done using the statistical package SPSS (Chicago, IL), version 17 . The chi-square test and Fisher exact test were used to examine the association between molecular marker expression and various clinicopathologic parameters. Plots of the disease-specific survival (DSS) according to marker expression were drawn using the Kaplan-Meier method, and statistical significance between survival curves was assessed by the log-rank test. DSS was determined from the date of surgery to the time of glioma death. The multivariate analysis was carried out using the Cox proportional hazards model. All the clinic pathological indexes and miR-214 expression were entered into the Cox regression analysis. The significance level used was $\mathrm{P}<0.05$.

\section{Results}

Clinocopathologic variables. Demographic, clinical, and histopathologic variables of each included subjects are recorded. The median age was 67 years (range, $14-85$ years) and about half of patients were male (52.6\%). The glioma cases comprised14 low-grade pilocyticastrocytomas (WHO I), 30 


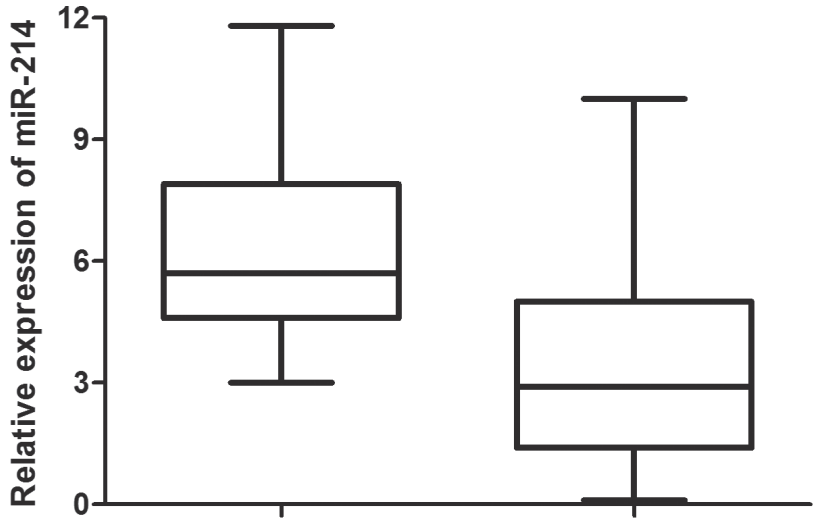

Glioma tissue Non-neoplastic brain tissues

Figure 1. MiR-214 expression in glioma and adjacent non-neoplatic brain tissues. MiRNA were detected by quantitative real-time polymerase chain reaction (qRT-PCR) analysis. MiR-214 expression was detected in 175 pairs of glioma and adjacent non-neoplastic brain tissues normalized to U6. As shown in Figure 1, we found that the expression of miR-214 was distinctly increased in glioma tissues compared to non-neoplastic brain tissues (mean $\pm \mathrm{SD}: 6.3 \pm 2.3$ vs. $3.4 \pm 2.2, \mathrm{P}<0.001$ ).

Table 1. Correlation of miR-214 expression with clinicopathological features of gliomas

\begin{tabular}{|c|c|c|c|c|}
\hline \multirow{2}{*}{$\begin{array}{l}\text { Clinicopathological } \\
\text { features }\end{array}$} & \multirow{2}{*}{$\begin{array}{l}\text { No. of } \\
\text { cases }\end{array}$} & \multicolumn{2}{|c|}{ miR-214 expression } & \multirow{2}{*}{$P$} \\
\hline & & High & Low & \\
\hline \multicolumn{5}{|l|}{ Mean age (year) } \\
\hline$<45$ & 95 & 45 & 50 & \multirow{2}{*}{0.261} \\
\hline$\geq 45$ & 80 & 36 & 44 & \\
\hline \multicolumn{5}{|l|}{ Gender } \\
\hline Male & 93 & 51 & 42 & \multirow{2}{*}{0.209} \\
\hline Female & 82 & 39 & 43 & \\
\hline \multicolumn{5}{|l|}{ WHO grade } \\
\hline WHO I & 14 & 4 & 10 & \multirow{4}{*}{0.027} \\
\hline WHO II & 30 & 11 & 19 & \\
\hline WHO III & 52 & 26 & 26 & \\
\hline WHO IV & 79 & 49 & 30 & \\
\hline \multicolumn{5}{|l|}{ KPS } \\
\hline$\geq 80$ & 102 & 67 & 37 & \multirow{2}{*}{0.047} \\
\hline$<80$ & 73 & 37 & 36 & \\
\hline \multicolumn{5}{|l|}{ Tumor size (cm) } \\
\hline$\geq 6$ & 122 & 71 & 51 & \multirow{2}{*}{0.210} \\
\hline$<6$ & 53 & 32 & 31 & \\
\hline \multicolumn{5}{|l|}{ Extent of resection } \\
\hline Total resection & 139 & 75 & 64 & \multirow{2}{*}{0.508} \\
\hline Partial resection & 36 & 20 & 16 & \\
\hline \multicolumn{5}{|l|}{ Adjuvant treatment } \\
\hline Radiotherapy & 32 & 20 & 12 & \multirow{3}{*}{0.901} \\
\hline Chemotherapy & 6 & 3 & 4 & \\
\hline $\begin{array}{l}\text { Radiotherapy combined } \\
\text { with Chemotherapy }\end{array}$ & 27 & 15 & 12 & \\
\hline
\end{tabular}

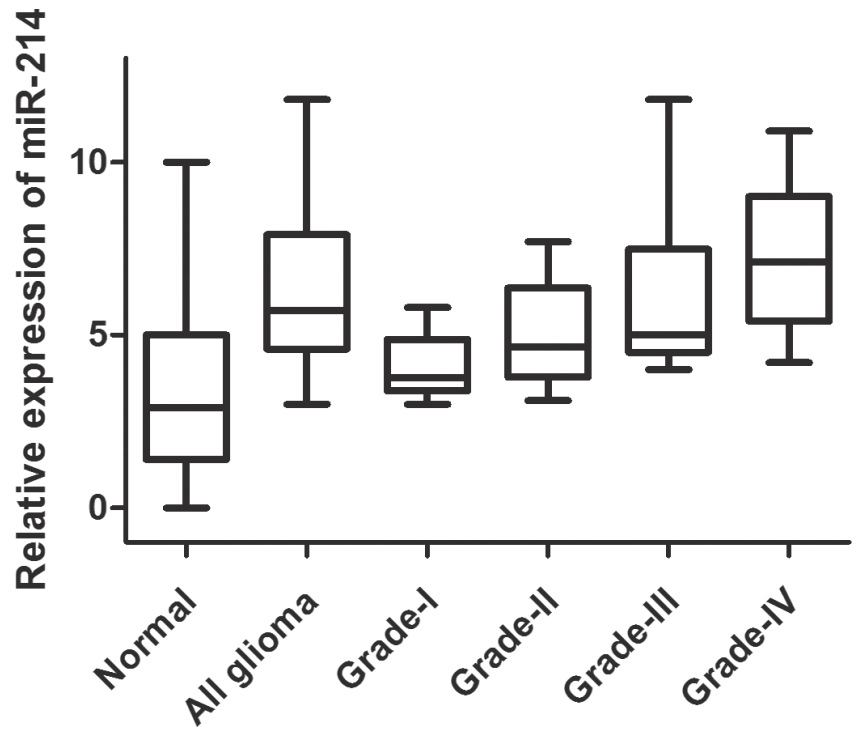

Figure 2. MiR-214 expression by different tumor stages. MiR-214 expression in 175 pairs of glioma and adjacent non-neoplatic brain tissues detected by quantitative real-time polymerase chain reaction (qRT-PCR) analysis according to different tumor stages (normal, all cancer and grade I-IV). diffuse astrocytomas (WHO II), 131 high-grade gliomas [52 anaplasia astrocytomas (WHO III), and 58 primary glioblastomas (WHO IV)]. A total of 32 patients had received chemotherapy, 6 received radiotherapy and 27 received radiotherapy combined with chemotherapy prior to surgery.

MiR-214 upregulation in human glioma tissues. MiR-214 expression was detected in 175 pairs of glioma and adjacent non-neoplastic brain tissues normalized to U6. As shown in Figure 1, we found that the expression of miR-214 was distinctly increased in glioma tissues compared to non-neoplastic brain tissues (mean $\pm \mathrm{SD}: 6.3 \pm 2.3$ vs. $3.4 \pm 2.2, \mathrm{P}<0.001$ ). In addition, miR-214 expression in high-grade (III-IV; $6.8 \pm 1.9$ ) and lowgrade (I-II; $4.5 \pm 1.3$ ) glioma were both significantly higher than that in non-neoplastic brain tissues $(3.4 \pm 2.2 ; \mathrm{P}<0.001$ and 0.01 , respectively, Figure 2 ). There was also a significant difference in miR-214 expression between high-grade (III-IV) and low -grade (I-II) glioma tissue specimens ( $\mathrm{P}=0.001$, Figure 2$)$.

Upregulation of miR-214 associated with advanced clinicopathological features of glioma. The associations of miR-214 expression with various clinicopathological parameters of glioma tissues were analyzed. The median of miR-214 expression levels in all 175 patients with glioma was 6.3. The patients were divided into two groups according to their expression levels of miR-214, using its median as a cutoff: high miR-214 expression group $(\mathrm{n}=90$; mean \pm SD: $8.0 \pm 2.6)$ and low miR-214 expression group $(\mathrm{n}=85$; mean \pm SD: $4.5 \pm 1.7)$. As shown in Table 1, miR-214 was significantly upregulated in glioma patients with advanced clinical stage $(\mathrm{P}=0.027)$ as compared to those with low clinical stage. The upregulation of miR-214 was associated with a higher KPS score $(P=0.047)$. 
No significant difference was observed between the expression of miR-214 and patients' age, gender, tumor size, extent of resection and adjuvant treatment.

Upregulation of miR-214 confers poor prognosis in patients with glioma. Using the Kaplan-Meier method and logrank test, the overall survival of glioma patients with high miR-214 expression was significantly shorter than those with low miR-214 expression $(\mathrm{P}<0.001$; Figure 3a). Besides, the survival benefits were also found in those with lower clinical stage (WHO grade I-II, $\mathrm{P}=0.001$ ) and higher clinical stage (WHO grade III-IV, $\mathrm{P}<0.001$ ).

Multivariate Cox regression analysis enrolling above-mentioned significant parameters revealed that miR-214 expression (relative risk [RR] 4.78; 95\% CI 3.10-7.37; $\mathrm{P}<0.001$ ), WHO grade (RR 3.8; 95\% CI 1.3-6.4; $\mathrm{P}=0.01$ ) and KPS score (RR 2.5; $95 \%$ CI 1.2-5.3; $\mathrm{P}=0.02$ ) were independent prognostic markers for overall survival of patients with glioma (Table 2). While the age, gender, WHO grade, tumor size, extent of resection, adjuvant treatment and type of adjuvant treatment were not independent prognostic markers $(\mathrm{P}>0.05)$.

We further analyzed the 5 year overall survival of miR-214 expression in selective patient subgroups stratified according to the WHO classification. MiR-214 expression was significantly associated with poor 5 year overall survival in glioma patients with high pathological grades (for grade III IV: $\mathrm{P}<0.001$; Table 3 ) and as significant as in patients with low pathological grades (for grade I II: $\mathrm{P}=0.04$; Table 3 ). However, the association between miR-214 expression with 5 year overall survival was

Table 2. Univariate and multivariate analyses of different prognostic parameters in patients with gliomas by Cox regression analysis

\begin{tabular}{lccc}
\hline Parameter & Risk ratio & $\begin{array}{c}\text { 95\% confidence } \\
\text { interval }\end{array}$ & $\boldsymbol{P}$ \\
\hline Age & 1.3 & $0.67-2.16$ & 0.53 \\
Gender & 1.7 & $0.45-2.65$ & 0.45 \\
WHO grade & 3.8 & $1.3-6.4$ & $\mathbf{0 . 0 1}$ \\
KPS & 2.5 & $1.2-5.3$ & $\mathbf{0 . 0 2}$ \\
Tumor size & 1.9 & $0.9-5.3$ & 0.13 \\
Extent of resection & 2.8 & $0.8-4.2$ & 0.21 \\
Adjuvant treatment & 2.0 & $0.6-3.8$ & 0.36 \\
Type of adjuvant treatment & 2.1 & $0.5-4.5$ & 0.41 \\
miR-214 expression & 4.78 & $3.10-7.37$ & $<\mathbf{0 . 0 0 1}$ \\
\hline
\end{tabular}

Table 3. Subgroup log-rank analysis of miR-214 expression and prognosis in patients with different pathological grades

\begin{tabular}{lccc}
\hline WHO grade & $\begin{array}{c}\text { miR-214 expression } \\
\text { status }\end{array}$ & $\begin{array}{c}\text { 5 year overall sur- } \\
\text { vival (\%) }\end{array}$ & $\boldsymbol{P}$ \\
\hline \multirow{2}{*}{ I II } & High & 45.4 & \multirow{2}{*}{$\mathbf{0 . 0 4}$} \\
\multirow{2}{*}{ III $\sim$ IV } & Low & 82.5 & \\
& High & 15.8 & $<\mathbf{0 . 0 0 1}$ \\
\hline
\end{tabular}

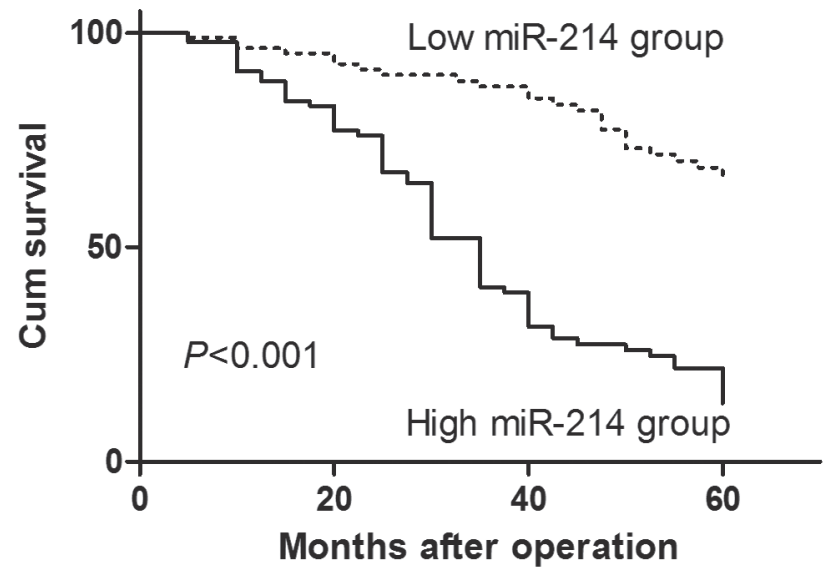

(a)

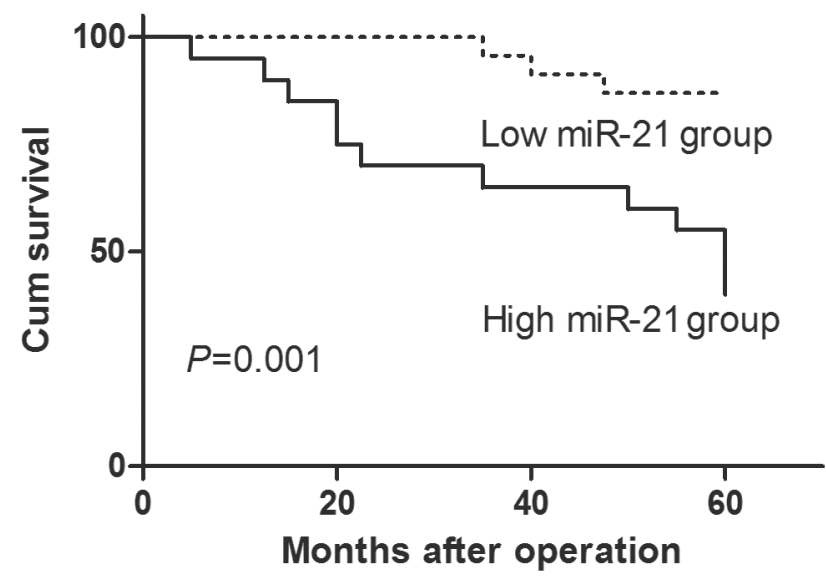

(b)

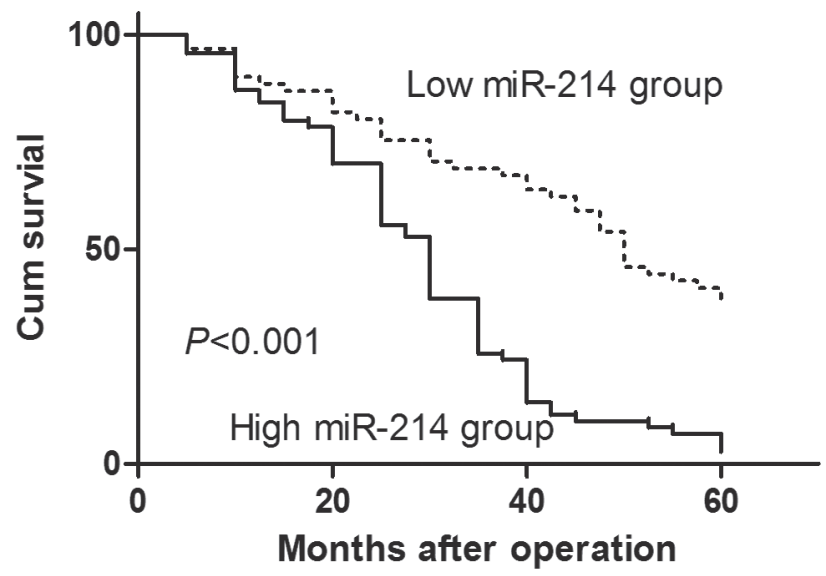

(c)

Figure 3. Kaplan-Meier survival curves for glioma patients with high or low expression of miR-214. (a) The 5-year overall survival rate of all 175 glioma patients with high or low miR-214 expression; (b) The 5-year overall survival rate of $\mathbf{4 4}$ glioma patients with low pathological grades (tumor stage I II) in high or low miR-21 expression group; (c) The 5-year overall survival rate of 131 glioma patients with advanced pathological grades (tumor stage III IV) in high or low miR-214 expression group 
more significant in the high pathological group compared with low pathology group.

\section{Discussion}

Recently, attentions have been focused on the role of microRNA regulation in essential mechanisms for cancer progression and metastasis, including proliferation, invasion, migration, angiogenesis and apoptosis. In human cancers, previous studies have been also shown that dysregulation of certain microRNAs are associated with clinical outcomes of several kinds of cancers [15-17]. A few reports even demonstrated that the expression profiling of microRNAs may be a more accurate method of classifying cancer subtypes than using the expression profiles of protein-coding genes [18].

In the present study, we confirmed that the expression level of miR-214 was increased in glioma tissues compared with the non-neoplastic brain tissues. Next, the Kaplan-Meier analysis revealed that glioma patients with high miR-214 expression tend to have poorer overall survival. In addition, the multivariate analysis clearly demonstrated that high miR-214 expression was a statistically significant risk factor affecting overall survival in glioma patients, suggesting that miR-214 upregulation in glioma is not only in a grade-dependent fashion, it is also a predictor of overall survival. Finally, subgroup analyses showed the significant prognostic value of miR-214 upregulation for glioma patients in those with low and high pathological grade.

There are kinds of miRNAs have been shown increased or decreased in glioma tissues or cell lines in previous studies. Qiu et al conducted a study to detect the abnormal expressed miRNAs in glioma. Their results found that high levels of miR326/miR-130a and low levels of miR-323/miR-329/miR-155/ miR-210 were significantly associated with long overall survival of glioblastoma patients, and also showed that high miR-326/ miR-130a and low miR-155/miR-210 were related with extended progression free survival [11]. In another study based on 168 cases glioma specimens and 21 normal control brain specimens, miR-650 was reported was related to World Health Organization grade and Karnofsky performance score for high expression was more frequently detected in glioma of high grade or low Karnofsky performance score [19]. Several studies are wanted in the future to detect the global differences between glioma and normal tissues and thus it could be concluded that whether miR-214 is a specified changed miRNA for giloma.

Our results mentioned above are similar to the results of the previous studies. Wang et al demonstrated that upregulated expression of microRNA-214 is linked to tumor progression and adverse prognosis in pediatric osteosarcoma [20]. In ovarian cancer, miR-214 was shown to induce cell survival and cisplatin resistance by targeting the 3 '-untranslated region (UTR) of PTEN to suppress its expression and resulting in the activation of the PI3K/Akt signaling pathway [21]. In cervical cancer, the ectopic expression of miR-214 could inhibit the proliferation, migration and invasive ability of HeLa cells by targeting MEK3, JNK1 and Plexin-B1 [22]. Studies have also reported that miR-214 contributes to the progression and metastasis of melanoma through the suppression of TFAP2C. $\mathrm{Xu}$ et al reported that the elevated expression of miR-214 was associated with chemoresistance and metastasis of ovarian cancer, and enforcing to increase expression of miR-214, whereas knockdown of miR-214 could decrease, ovarian cancer stem cell population and self-renewal as well as the Nanog level preferentially in wild-type p53 cell lines [23]. Schwarzenbach et al performed analyses on the preoperative serum samples of 102 patients with early breast cancer and a subset of 34 postoperative samples, as well as of 32 patients with benign breast disease and 53 healthy women. The results showed that serum miR-214 could discriminate malignant from benign tumors and healthy controls and increased miR-214 levels were associated with a positive lymph node status [24]. These examples illustrate the importance of the proper execution of miR-214 for maintenance of cellular homeostasis and the optimal performance of cellular processes and miR-214 expression is often perturbed in human cancer.

There are but some recent studies with contradictory results of this study. Zhao et al found that miR-124 level was downregulated in glioblastoma tissues. Moreever miR-214 was inversely regulated PPP1R13L expression in different glioblastoma cell lines. Recent studies showed that PPP1R13L was found to be a direct target of miR-124 in glioblastoma cells [25]. Wang et al published a study detecting the effect of miR-214 and UBC9 on the gilomas. Their results demonstrated that, the expression levels of miR-214 and UBC9 mRNA in glioma tissues were significantly downregulated and upregulated, when compared with normal brain tissues [26]. Their results are contradictory with this study. As we know, the effects of miR-214 are complex and the pathology of glioma was also manifold and unclear. Besides, the glioma is a combination of kinds of different pathological subgroup carcinomas, thus might be a source of the uncertainty. This study is based on a relative large amount of patients and a 5 years' follow-up, thus we think it would provide reliable meanings. To provide a stronger evidence of our conclusion, there is a need of more experimental studies to find the molecular and pathway mechanisms of miR-214 in glioma pathology.

Although the current study interestingly found the association of miR-214 up-regulation with the aggressive tumor progression and unfavorable prognosis in glioma, it was limited by its retrospective nature which led to our results being considered exploratory. Additionally, the molecular mechanisms and direct functional targets of miR-214 in glioma are still limited in this study and additional studies are required for the further recognition.

In conclusion, the results of this study showed that miR-214 was up-regulated in glioma tissues. The expression of miR214 was associated with the pathological stages of glioma. The results of 5-years follow-up showed that the expression level of miR-214 is a significant prognostic factor for patients with glioma. Our results demonstrated that miR-214 might be a potential biomarker of the prognostic outcome of glioma. The 
detailed molecular mechanism through which miR-214 worked in the glioma should be studied in the following studies.

\section{References}

[1] LEFRANC F, BROTCHI J, KISS R. Possible future issues in the treatment of glioblastomas: special emphasis on cell migration and the resistance of migrating glioblastoma cells to apoptosis. J Clin Oncol. 2005; 23: 2411-22. http://dx.doi. org/10.1200/JCO.2005.03.089

[2] FURNARI FB, FENTON T, BACHOO RM, MUKASA A, STOMMEL JM, et al. Malignant astrocytic glioma: genetics, biology, and paths to treatment. Genes Dev. 2007; 21: 2683-710. http://dx.doi.org/10.1101/gad.1596707

[3] SIEGEL MJ, FINLAY JL, ZACHAROULIS S. State of the art chemotherapeutic management of pediatric brain tumors. Expert Rev Neurother. 2006; 6: 765-79. http://dx.doi. org/10.1586/14737175.6.5.765

[4] HOLLAND EC. Gliomagenesis: genetic alterations and mouse models. Nat Rev Genet. 2001; 2: 120-9. http://dx.doi. org/10.1038/35052535

[5] LU J, GETZ G, MISKA EA, ALVAREZ-SAAVEDRA E, LAMB, et al. MicroRNA expression profiles classify human cancers. Nature. 2005; 435: 834-8. http://dx.doi.org/10.1038/nature03702

[6] CALIN GA, CROCE CM. MicroRNA signatures in human cancers. Nat Rev Cancer. 2006; 6: 857-66. http://dx.doi. org/10.1038/nrc1997

[7] KETTING RF, FISCHER SE, BERNSTEIN E, SIJEN T, HANNON GJ, et al. Dicer functions in RNA interference and in synthesis of small RNA involved in developmental timing in C. elegans. Genes Dev. 2001; 15: 2654-9. http://dx.doi. org $/ 10.1101 /$ gad. 927801

[8] CALIN GA, SEVIGNANI C, DUMITRU CD, HYSLOP T, $\mathrm{NOCH}$ E, et al. Human microRNA genes are frequently located at fragile sites and genomic regions involved in cancers. Proc Natl Acad Sci U S A. 2004; 101: 2999-3004. http://dx.doi. org/10.1073/pnas.0307323101

[9] JOHNSON SM, GROSSHANS H, SHINGARA J, BYROM $\mathrm{M}$, JARVIS R, et al. RAS is regulated by the let-7 microRNA family. Cell. 2005; 120: 635-47. http://dx.doi.org/10.1016/j. cell.2005.01.014

[10] HAYASHITA Y, OSADA H, TATEMATSU Y, YAMADA H, YANAGISAWA $\mathrm{K}$, et al. A polycistronic microRNA cluster, miR-17-92, is overexpressed in human lung cancers and enhances cell proliferation. Cancer Res. 2005; 65: 9628-32. http://dx.doi.org/10.1158/0008-5472.CAN-05-2352

[11] XIAO B, TAN L, HE B, LIU Z, XU R. MiRNA-329 targeting E2F1 inhibits cell proliferation in glioma cells. J Transl Med. 2013; 11: 172. http://dx.doi.org/10.1186/1479-5876-11-172

[12] LEE HK, BIER A, CAZACU S, FINNISS S, XIANG C, et al. MicroRNA-145 is downregulated in glial tumors and regulates glioma cell migration by targeting connective tissue growth factor. PLoS One. 2013; 8: e54652. http://dx.doi.org/10.1371/ journal.pone.0054652

[13] DERFOUL A, JUAN AH, DIFILIPPANTONIO MJ, PALANISAMY N, RIED T, et al. Decreased microRNA-214 levels in breast cancer cells coincides with increased cell proliferation, invasion and accumulation of the Polycomb Ezh2 methyltransferase. Carcinogenesis. 2011; 32: 1607-14. http://dx.doi. org $/ 10.1093 / \operatorname{carcin} / \operatorname{bgr} 184$

[14] ZHAO Z, TAN X, ZHAO A, ZHU L, YIN B, et al. microRNA214-mediated UBC9 expression in glioma. BMB Rep. 2012; 45: 641-6. http://dx.doi.org/10.5483/BMBRep.2012.45.11.097

[15] ALMEIDA MI, NICOLOSO MS, ZENG L, IVAN C, SPIZZO $\mathrm{R}$, et al. Strand-specific miR-28-5p and miR-28-3p have distinct effects in colorectal cancer cells. Gastroenterology. 2012; 142: 886-96 e9.

[16] YANG M, YAO Y, EADES G, ZHANG Y, ZHOU Q. MiR28 regulates Nrf2 expression through a Keap1-independent mechanism. Breast Cancer Res Treat. 2011; 129: 983-91. http://dx.doi.org/10.1007/s10549-011-1604-1

[17] FLAVIN RJ, SMYTH PC, LAIOS A, O‘TOOLE SA, BARRETT C, et al. Potentially important microRNA cluster on chromosome $17 \mathrm{p} 13.1$ in primary peritoneal carcinoma. Mod Pathol. 2009; 22: 197-205. http://dx.doi.org/10.1038/ modpathol.2008.135

[18] VOLINIA S, CALIN GA, LIU CG, AMBS S, CIMMINO A, et al. A microRNA expression signature of human solid tumors defines cancer gene targets. Proc Natl Acad Sci U S A. 2006; 103: 2257-61. http://dx.doi.org/10.1073/pnas.0510565103

[19] SUN B, PU B, CHU D, CHU X, LI W, et al. MicroRNA-650 expression in glioma is associated with prognosis of patients. J Neurooncol. 2013; 115: 375-80. http://dx.doi.org/10.1007/ s11060-013-1243-y

[20] WANG Z, CAI H, LIN L, TANG M. Upregulated expression of microRNA-214 is linked to tumor progression and adverse prognosis in pediatric osteosarcoma. Pediatr Blood Cancer. 2013.

[21] YANG H, KONG W, HE L, ZHAO JJ, O'DONNELL JD, et al. MicroRNA expression profiling in human ovarian cancer: miR-214 induces cell survival and cisplatin resistance by targeting PTEN. Cancer Res. 2008; 68: 425-33. http://dx.doi. org/10.1158/0008-5472.CAN-07-2488

[22] QIANG R, WANG F, SHI LY, LIU M, CHEN S, et al. Plexin$\mathrm{B} 1$ is a target of miR-214 in cervical cancer and promotes the growth and invasion of HeLa cells. Int J Biochem Cell Biol. 2011; 43: 632-41. http://dx.doi.org/10.1016/j.biocel.2011.01.002

[23] BAR-ELI M. Searching for the ,melano-miRs': miR-214 drives melanoma metastasis. EMBO J. 2011; 30: 1880-1. http:// dx.doi.org/10.1038/emboj.2011.132

[24] SCHWARZENBACH H, MILDE-LANGOSCH K, STEINBACH B, MULLER V, PANTEL K. Diagnostic potential of PTEN-targeting miR-214 in the blood of breast cancer patients. Breast Cancer Res Treat. 2012; 134: 933-41. http:// dx.doi.org/10.1007/s10549-012-1988-6

[25] ZHAO WH, WU SQ, ZHANG YD. Downregulation of miR124 promotes the growth and invasiveness of glioblastoma cells involving upregulation of PPP1R13L. Int J Mol Med. 2013; 32: 101-7.

[26] WANG S, JIAO B, GENG S, MA S, LIANG Z, et al. Combined aberrant expression of microRNA-214 and UBC9 is an independent unfavorable prognostic factor for patients with gliomas. Med Oncol. 2014; 31: 767. http://dx.doi.org/10.1007/s12032-013$\underline{0767-5}$ 